Final Report

\title{
Novel Large Area High Resolution Neutron Detector for the Spallation Neutron Source
}

DOE SBIR Phase II Grant No. DE-FG02-05ER84251

Principal Investigator: Jeffrey L. Lacy, Ph.D.

Recipient: Proportional Technologies, Inc., Houston, TX 77054

$\underline{\text { To }}$

Helen M. Kerch

DOE Project Officer

helen.kerch@science.doe.gov

sbir-sttr@science.doe.gov

From

Jeffrey L. Lacy, Ph.D.

President

Proportional Technologies, Inc.

8022 El Rio St.

Houston TX 77054

(713) 747-7324

jlacy@proportionaltech.com

Date: April 10, 2009 


\section{EXECUTIVE SUMMARY}

Neutron scattering is a powerful technique that is critically important for materials science and structural biology applications. The knowledge gained from past developments has resulted in far-reaching advances in engineering, pharmaceutical and biotechnology industries, to name a few. New facilities for neutron generation at much higher flux, such as the SNS at Oak Ridge, $\mathrm{TN}$, will greatly enhance the capabilities of neutron scattering, with benefits that extend to many fields and include, for example, development of improved drug therapies and materials that are stronger, longer-lasting, and more impact-resistant.

In order to fully realize this enhanced potential, however, higher neutron rates must be met with improved detection capabilities, particularly higher count rate capability in large size detectors, while maintaining practicality. We have developed a neutron detector with the technical and economic advantages to accomplish this goal. This new detector has a large sensitive area, offers 3D spatial resolution, high sensitivity and high count rate capability, and it is economical and practical to produce. The proposed detector technology is based on ${ }^{10} \mathrm{~B}$ thin film conversion of neutrons in long straw-like gas detectors. A stack of many such detectors, each 1 meter in length, and $4 \mathrm{~mm}$ in diameter, has a stopping power that exceeds that of ${ }^{3} \mathrm{He}$ gas, contained at practical pressures within an area detector. With simple electronic readout methods, straw detector arrays can provide spatial resolution of $4 \mathrm{~mm}$ FWHM or better, and since an array detector of such form consists of several thousand individual elements per square meter, count rates in a $1 \mathrm{~m}^{2}$ detector can reach $2 \times 10^{7} \mathrm{cps}$. Moreover, each individual event can be timetagged with a time resolution of less than $0.1 \mu \mathrm{sec}$, allowing accurate identification of neutron energy by time of flight. Considering basic elemental cost, this novel neutron imaging detector can be commercially produced economically, probably at a small fraction of the cost of ${ }^{3} \mathrm{He}$ detectors.

In addition to neutron scattering science, the fully developed base technology can be used as a rugged, low-cost neutron detector in area monitoring and surveying. Radiation monitors are used in a number of other settings for occupational and environmental radiation safety. Such a detector can also be used in environmental monitoring and remote nuclear power plant monitoring. For example, the Department of Energy could use it to characterize nuclear waste dumps, coordinate clean-up efforts, and assess the radioactive contaminants in the air and water. Radiation monitors can be used to monitor the age and component breakdown of nuclear warheads and to distinguish between weapons and reactor grade plutonium. The UN's International Atomic Energy Agency (IAEA) uses radiation monitors for treaty verification, remote monitoring, and enforcing the non-proliferation of nuclear weapons. As part of treaty verification, monitors can be used to certify the contents of containers during inspections. They could be used for portal monitoring to secure border checkpoints, sea ports, air cargo centers, public parks, sporting venues, and key government buildings. Currently, only $2 \%$ of all sea cargo shipped is inspected for radiation sources. In addition, merely the presence of radiation is detected and nothing is known about the radioactive source until further testing. The utilization

of radiation monitors with neutron sensitivity and capability of operation in hostile port environments would increase the capacity and effectiveness of the radioactive scanning processes. 


\section{ACCOMPLISHMENTS vs. OBJECTIVES}

The objectives of the project were as follows:

Objective 1: The design and adjustment of the front end amplifier will be optimized to achieve maximum integral count rates without loss of spatial resolution.

Actual accomplishment: All goals set in Objective 1 have been met. Fully operational amplifier boards for all 22 detectors and a complete data digitization system have been developed.

Objective 2: Depending on the peak rate reached with a single amplifier in Objective 1 a decoding scheme employing smaller groupings of straws will be developed if needed to provide an integral count rate performance of $2 \times 10^{7}$ in a $1 \mathrm{~m}^{2}$ detector.

Actual accomplishment: All goals set in Objective 2 have been met. Major improvements in integral count rate performance were achieved with the re-design of the front electronics and signal conditioning in the CAMAC module, under Objective 1, and thus module segmentation into less than 50 straw modules was not necessary.

Objective 3: A robust aluminum housing will be developed to facilitate housing of 66 straw detector modules with 50 straws of length 1 meter in each module in a 3 deep configuration producing a $1 \mathrm{~m}^{2}$ detector active area. The housing will facilitate accurate close packing of modules with no more than $1 \mathrm{~mm}$ gap between adjacent units and will accommodate uniform distribution of the inter-module gaps uniformly through the detector volume in order to prevent image artifact. Each module will be self supporting in a vertical orientation and electrically isolated from adjacent units by the small gas gap which will be purged with $\mathrm{N}_{2}$ or other gas to provide electrical isolation of modules one from another while employing minimal neutron scattering material. The housing will accommodate easy removal and replacement of individual 50 straw modules together with amplifier boards through access at the top of the detector housing.

Actual accomplishment: All goals set in Objective 3 have been met. The proposed housing was completed and populated with 22 working detector modules, as described in detail below.

Objective 4: Alternative gas mixtures known to provide somewhat improved detector lifetime and safer (nonflammable) operation will be evaluated and an optimal mixture for this application will be chosen.

Actual accomplishment: All goals set in Objective 4 have been met. The optimal gas mixture was determined to be $90 \% \mathrm{Ar}, 10 \% \mathrm{CO}_{2}$. The mixture provides stable operation for detector bias up to $1200 \mathrm{~V}$, is nonflammable, and has excellent lifetime. 
Objective 5: Comprehensive lifetime testing will be carried out with detectors subjected to high rate neutron irradiation to predict long term performance in actual SNS application.

Actual accomplishment: Based on recent extensive research in the high energy physics field it has been conclusively demonstrated that mixtures of Argon with $\mathrm{CO}_{2}$ provide nearly indefinite detector lifetime and are far superior to any other tested mixture.

Objective 6: A large number of detector modules will be constructed with a goal of fully populating the 66 detector positions in the housing developed in Objective 3.

Actual accomplishment: 22 detector modules were successfully completed and tested. They have been integrated into the housing of Objective 3 to form a fully operational square meter panel detector.

Objective 7: Electronic amplifier boards of optimized design will be fabricated for all of the detector modules of 6 and digital electronics required to readout these modules will be procured.

Actual accomplishment: All goals set in Objective 7 have been met. Fully operational amplifier boards for all 22 detectors and a complete data digitization system have been developed. All components have been combined to form a fully operational square meter panel detector.

Objective 8: All detectors and amplifier boards will be integrated into the housing of Objective 3 and the entire $1 \mathrm{~m}^{2}$ detector will be deployed at an appropriate test facility and thoroughly tested to establish integral and differential rate performance, spatial resolution, sensitivity, gamma ray rejection, and gamma random rate tolerance.

Actual accomplishment: Due to limited resources, we were unable to evaluate the performance of the fully integrated system at a remote testing facility. In-house testing was limited to the use of a moderated ${ }^{252} \mathrm{Cf}$ source. However, in a number of phantom studies, we were able to verify that the fully integrated, square-meter panel detector was operational and stable, and that 3D encoding of neutron interactions was successful. 


\section{PROJECT ACTIVITIES SUMMARY}

\section{Activities for Task 1: Front-end amplifier}

The objective for Task 1 was the design and adjustment of the front-end amplifier and its optimization to achieve maximum integral count rate without the loss of spatial resolution, including comprehensive testing in the high rate neutron beam at the reactor test facility located at Texas A\&M University.

The front end analogue circuitry was completely redesigned as follows:

1. The amplifier was re-configured as two modular components - a current sensitive preamplifier module in the detector housing, and a standard CAMAC unit containing a shaper, a summing amplifier, a delay line clipping circuit and discriminator circuits (postamplifier module). Fig. 1 shows the new pre-amplifier board connected to a 50-straw detector. With the much smaller board size (only $30 \%$ of the previous length), integration of pre-amplifiers and all 66 detectors in the entire $1 \mathrm{~m}^{2}$ detector housing is much more convenient and the overall size of the housing is reduced substantially. Fig. 2 shows the functional schematic diagram of electronics for the complete system. The analog timing and charge signals coming out of the post-amp will pass directly to a customized digitizer module next to it, which converts amplitude and timing signals to 12 bit digital values and outputs them as a serial data stream with the Fast Ethernet protocol. These streams from a large number of digitizers are then combined into a single stream using an Ethernet Hub and passed to a PC or PC cluster.

2. Pre-amplifier circuitry was re-designed to achieve optimal performance. A non-inverting operational amplifier configuration was utilized, which because of the more proper termination of the decoding delay line eliminated reflections occurring at the delay line ends. Shaper circuitry was also re-designed with a custom-made CR-200 Gaussian shaping amplifier (Cremat Inc., Watertown, MA), with a shaping time constant of 40 nsec. The shaper filtered much of the noise from the signal of interest and provided a quickly restored baseline. A delay line clipping circuit was added following the shaper employing a high quality lumped constant delay line (TF300-5, Rhombus Industries Inc., Santa Ana, CA). This circuit further improved baseline restoration. Overall a factor of 6 improvement in count rate capability was measured by observation of pedestal broadening in response to increasing rate. Insignificant baseline offsets were observed for count rate up to $150,000 \mathrm{cps}$ on a single 50 -straw detector module. Thus we expect at least a factor of 4 increase in count rate capability increasing the projected $1 \mathrm{~m}^{2}$ detector integral count rate from $1 \times 10^{7}$ to $4 \times 10^{7} \mathrm{cps}$. This is a very important improvement increasing the capability almost to the level desired by SNS without having to segment detectors into smaller straw groups which both increases cost and complexity. This improvement required a substantial redesign of the complete front end electronic circuitry and development of two new circuit boards. 


\section{Activities for Task 2: Straw grouping}

The main objective of this task was to implement a new decoding scheme with smaller straw groupings as to provide an integral count rate performance of $2 \times 10^{7} \mathrm{cps}$ in a $1 \mathrm{~m}^{2}$ detector. Major improvements in integral count rate performance were achieved with the re-design of the front electronics and signal conditioning in the CAMAC module, as discussed above. With this optimal design, we expect at least a factor of 4 increase in count rate capability, increasing the projected $1 \mathrm{~m}^{2}$ detector integral count rate from $1 \times 10^{7}$ to $4 \times 10^{7} \mathrm{cps}$. With this improved performance, module segmentation into less than 50 straw modules is not required while still achieving a 2 fold improvement over the milestone.

\section{Activities for Task 3: Straw Module Housing}

A robust aluminum housing has been developed to facilitate housing of the 66 straw detector modules required for a sensitive area of $1 \mathrm{~m}^{2}$. Modules are placed in a staggered 3 deep configuration, allowing accurate close packing with a precise $1 \mathrm{~mm}$ gap between adjacent detectors. This placement prevents image artifacts and allows the electrical isolation required between detector modules. Detectors are positioned and supported in a vertical orientation through the use of PC board alignment pins, as shown in Fig. 3, and a precision machined Ultem 1000 alignment structure permanently mounted at the bottom of the housing, shown in Fig. 4.

Fig. 5 shows the 1 meter detector panel populated with 22 detector modules for testing. Modules with amplifiers installed as shown in Fig. 3 are slipped into the housing from the top. Once the amplifier board reaches the bottom of the housing it will be directed with alignment pins, allowing it to fall into place in the lower support structure. In this scheme the modules rest lightly against the bottom positioning plate which sets the vertical position of each module. The upper frame insert merely provides transverse positioning and rests lightly on the detector end boards.

\section{Activities for Task 4: Gas mixtures}

The main goal of this task was to evaluate alternative gas mixtures known to provide somewhat improved detector lifetime and safer (nonflammable) operation. In particular, different mixtures of argon/carbon dioxide $\left(\mathrm{Ar} / \mathrm{CO}_{2}\right)$ were evaluated and compared against the $\mathrm{P}-10$ (90\% Ar, 10\% $\mathrm{CH}_{4}$ ) mixture used previously. $\mathrm{CO}_{2}$ is non-flammable and has been shown to improve the ageing of gaseous detectors used at CERN.

We measured the gas multiplication factor, saturation, and stability properties of the different mixtures, in a single, one-meter long straw detector, identical to the straw detectors making up the large panel. The straw was $4 \mathrm{~mm}$ in diameter, with an aluminum wall cathode, and a $20 \mu \mathrm{m}$ anode wire tensioned to $31 \mathrm{~g}$. Gases were mixed on the fly, using flowmeters. A ${ }^{55} \mathrm{Fe}$ X-ray source with a characteristic energy of $5.9 \mathrm{keV}$ was used to ionize the gas. Signals were read out with a charge sensitive amplifier directly connected to the anode.

Fig. 6 shows the gas multiplication factor measured for the mixtures indicated, as a function of the applied potential. The $90 \% \mathrm{Ar} / 10 \% \mathrm{CO}_{2}$ mixture results in a higher 
multiplication factor compared to P-10. The desired operating gain of about 17,000, can be achieved at $1123 \mathrm{~V}$ with the $\mathrm{Ar} / \mathrm{CO}_{2}$ mixture, and at $1150 \mathrm{~V}$ with $\mathrm{P}-10$. Other mixtures require a higher potential since their gain curve is lower. The $\mathrm{Ar} / \mathrm{CO}_{2}$ ratio that results in the same gain as $\mathrm{P}-10$ is $82 / 18$.

We collected ${ }^{55} \mathrm{Fe}$ energy spectra in order to gauge the stability properties of each mixture. Fig. 7 shows spectra for P-10 and for $82 \%$ Ar $/ 18 \% \mathrm{CO}_{2}$. Saturation for both gas mixtures starts at around $1250 \mathrm{~V}$. That's $100 \mathrm{~V}$ over the operating point. Above $1150 \mathrm{~V}$, the $\mathrm{Ar} / \mathrm{CO}_{2}$ gain increases faster (steeper slope) than $\mathrm{P}-10$, but the $\mathrm{Ar} / \mathrm{CO}_{2}$ spectra appear more symmetrical at the higher gains.

\section{Activities for Task 5: Lifetime testing}

Based on recent extensive research in the high energy physics field it has been conclusively demonstrated that mixtures of Argon with $\mathrm{CO}_{2}$ provide nearly indefinite detector lifetime and are far superior to any other tested mixture. We therefore have extensively evaluated the mixture, 90\% Argon, $10 \% \mathrm{CO}_{2}$ and found that performance was in all respects superior to that of our initial $90 \%$ Argon, $10 \% \mathrm{CH}_{4}$ mixture. Higher gain is achieved at lower voltage and higher ultimate breakdown potential is at the same time achieved.

Based on the reputed lifetime performance of the new mixture, 90\% Argon, $10 \% \mathrm{CO}_{2}$ it was decided to move the priority of extensive lifetime testing down the list and evaluate lifetime as part of actual final detector operation in high rate neutron beams.

\section{Activities for Task 6: Straw Module Fabrication}

A major accomplishment achieved during Phase II was the development of an enriched boron carbide coating process. Analogous to this project is one granted to us by the Defense Threat Reduction Agency (DTRA) for the development of a large area monitor for passive, long range detection of neutron and gamma rays. This detector uses similar 50 straw 1 meter long modules, absent spatial resolution capability and can detect and localize remote neutron and gamma sources in the environment at great distances. Because this device requires enriched boron coated straws, substantial development of an enriched coating method was successfully achieved. Coating runs utilizing enriched boron were completed and straw material was later fabricated.

Sections from enriched and natural (non-enriched) coated material of equal length were fabricated into single, independent straw detectors and positioned in parallel $1 \mathrm{~cm}$ apart in a support fixture for performance evaluations, as shown in Fig. 8. Each of the straw detectors were tested for thermal neutron detection by operating both with the same set of electronics while within the same neutron field, generated by a small ${ }^{252} \mathrm{Cf}$ source and plastic thermalizer. The flux was characterized with a commercially available $\mathrm{BF}_{3}$ counter. The enriched straw material produced greater than six times increased sensitivity over the natural boron straw. This milestone provides a quantum leap enhancement in our neutron straw technologies with increased stopping power and detection efficiency.

With this major development, our priority shifted from completion of the 66 modules to 22 modules, which is enough to fully populate a single layer in the $1 \mathrm{~m}^{2}$ detector and provides ample detectors for thorough conceptual testing. This shift also allows generating fewer detectors 
which will become obsolete when the enriched coated modules are completed. All 22 modules having natural boron coating were successfully fabricated and tested following our design specifications and assembly methods. Each module has been tested for gas tightness, wire tension high voltage stability and gas gain uniformity.

Another important accomplishment has been development of very accurate measurements of the $\mathrm{B}_{4} \mathrm{C}$ coating thickness using cold neutron depth profiling at the NIST Center for Neutron Research. This technique provides quantitative depth profile measurements in solids for certain elements. A neutron beam illuminates our sample producing monoenergetic charged particles. By measuring the energy which the isotropically emitted particle retains upon leaving the sample, determined by the depth at which the interaction took place, depth profiles are acquired by deconvolution of the measured charged particle energy spectra, as illustrated in Fig. 9. Preliminary test samples showed our $\mathrm{B}_{4} \mathrm{C}$ coating thickness to be very uniform over the three initial production runs.

\section{Activities for Task 7: Electronic Readout Assembly}

The objective for Task 7 was the fabrication of electronic amplifier boards of optimized design for all of the detector modules of Task 6 and the procurement of digital electronics required to readout these modules.

$100 \%$ of the electronics necessary for 22 detector modules have been fabricated and tested, including several sets of spares. This includes more than 60 sub-module preamplifier boards, 11 signal conditioning modules and 11 signal digitization modules complete with CAMAC crate mechanics and gigabit Ethernet throughput to the controlling computer as well as all cables, harnesses, EMF shielding, high voltage and DC power supplies, as shown in Fig. 10.

\section{Activities for Task 8: System Integration \& Testing}

A detector panel with an active area of 1 square meter has been fully assembled, as shown in Fig. 5 , and has been tested successfully for basic operation. The panel consists of 22 detector modules, of the dimensions discussed above, and incorporates all required electronics for 3D readout. A fully operational, custom-designed data acquisition system has also been assembled and tested for basic operation. In particular, the integrated system consists of the following components:

- 22 detector modules

- aluminum housing

- Front-end electronics for detector signal readout

- Data acquisition system, including a CAMAC signal conditioning system (shaper, delay line clipping), ADCs, and TDCs.

- High voltage power supply for biasing the detectors, and low voltage supplies for powering the electronic components

- Computer and Ethernet switch

- Data acquisition and data analysis software 
The integrated system was tested as follows:

- All preamplifiers, CAMACs, ADCs/TDCs were individually tested with data acquisition and analysis software written in C++ and Matlab.

- All modules were operational at a bias voltage of $1150 \mathrm{~V}$, and with the $\mathrm{Ar} / \mathrm{CO}_{2}$ gas mixture.

- All detector modules were successfully tested for straw decoding with flood irradiation from a ${ }^{252} \mathrm{Cf}$ neutron source.

- Different tests for 2D imaging with $1 \mathrm{~m}^{2}$ panel of 22 detector modules were completed as described below.

The modules were operated together as a single detector, placed in front of a $6.1 \mu \mathrm{g}{ }^{252} \mathrm{Cf}$ source. The source was placed inside a polyethylene cylinder ( 4 inch diameter), 1 meter above ground and 2.2 meters from the detector panel.

Spatial resolution was evaluated in imaging studies, using a number of phantoms. Phantoms made from borated aluminum, plastic, or other neutron scattering/absorbing material were machined in-house. In one test an acrylic letter target ( $\mathrm{H}$ areal density: $90 \mathrm{mg} / \mathrm{cm}^{2}, 98.7 \%$ attenuation) was attached to the front panel of the detector housing, as shown in Figure 11a. Letters were $9.5 \mathrm{~mm}$ thick and $55 \mathrm{~mm}$ tall. Figure $11 \mathrm{~b}$ shows the image constructed with 20 million neutron events.

In another test, a collection of random objects of different material composition, shape and thickness, was used as depicted in Figure 12a. Figure 12b shows the image constructed with 13 million neutron events. The reconstructed objects at bottom of image are not perfectly straight, due to the insufficient "module-to-module" calibration in preliminary study. 


\section{PRODUCTS DEVELOPED}

a. Publications and conference papers

$\circ$ J.L. Lacy, A. Athanasiades, N.N. Shehad, C.S. Martin, and L. Sun. "Performance of 1 Meter Straw Detector for High Rate Neutron Imaging", IEEE 2006 Nuclear Science Symposium Conference Record, vol. 1 (2006), pp. 20-26.

- A. Athanasiades, N.N. Shehad, C.S. Martin, L. Sun and J.L. Lacy. "Straw Detector for High Rate, High Resolution Neutron Imaging”, IEEE 2005 Nuclear Science Symposium Conference Record, vol. 2 (2005), pp. 623-627.

- J.L. Lacy, A. Athanasiades, N.N. Shehad, R.A. Austin, and C.S. Martin. "Novel Neutron Detector for High Rate Imaging Applications", IEEE 2002 Nuclear Science Symposium Conference Record, vol 1 (2002), pp. 392-396.

b. Web sites

Considerable details on the design, development and performance of the neutron imaging detector have been published on the company's website, at the following address:

http://www.proportionaltech.com/new site/index.php?option $=$ com content\&view $=$ article\&id $=6$ $\underline{2 \& \text { Itemid }=73}$

A number of references, including the publications listed above, are available as downloadable DPF documents.

c. Technologies/techniques

The technologies and techniques developed during the course of this project include:

- Deposition of ${ }^{10} \mathrm{~B}$-enriched boron carbide $\left({ }^{10} \mathrm{~B}_{4} \mathrm{C}\right)$ onto aluminum film in a manner that achieves uniform thickness, and accuracy to the level of $0.1 \mu \mathrm{m}$.

- Measurement of the thickness of the ${ }^{10} \mathrm{~B}_{4} \mathrm{C}$ material deposited onto aluminum film, with a resolution better than $0.1 \mu \mathrm{m}$.

d. Inventions/Patents

A patent has been issued through the US Patent and Trademark Office to Proportional Technologies, Inc. on 2/21/2006 titled "Boron Coated Straw Neutron Detector" with the award number 7,002,159 B2. 
e. Instruments/equipment

The instruments/equipment acquired or developed during the course of the project include:

- Straw detectors

- Front-end electronics for reading out straw detectors

- Data acquisition system for signal processing

- Personal computer

- $\quad{ }^{252}$ Cf neutron source 


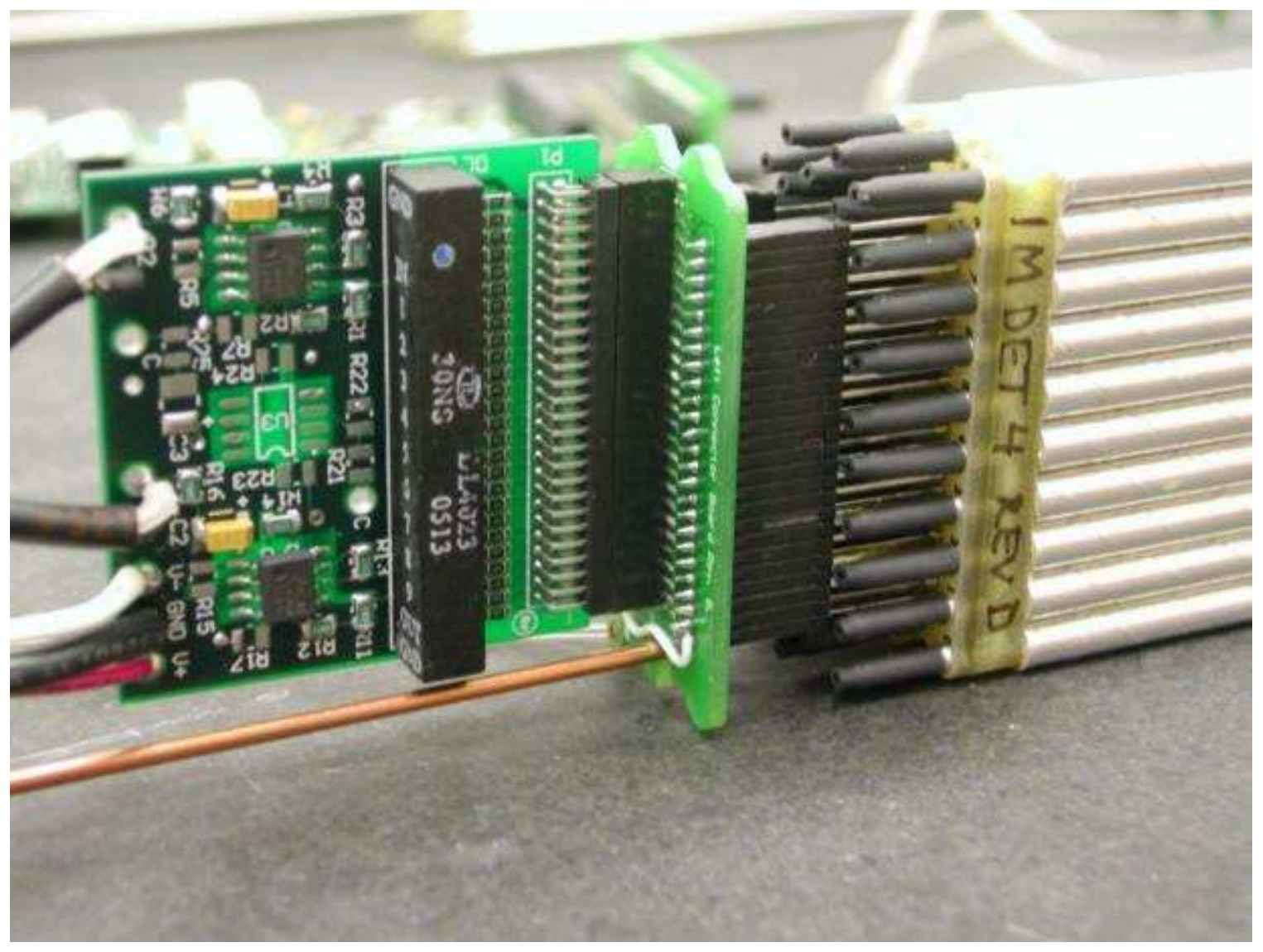

Fig. 1. New pre-amplifier board connected to a 50-straw detector module. 


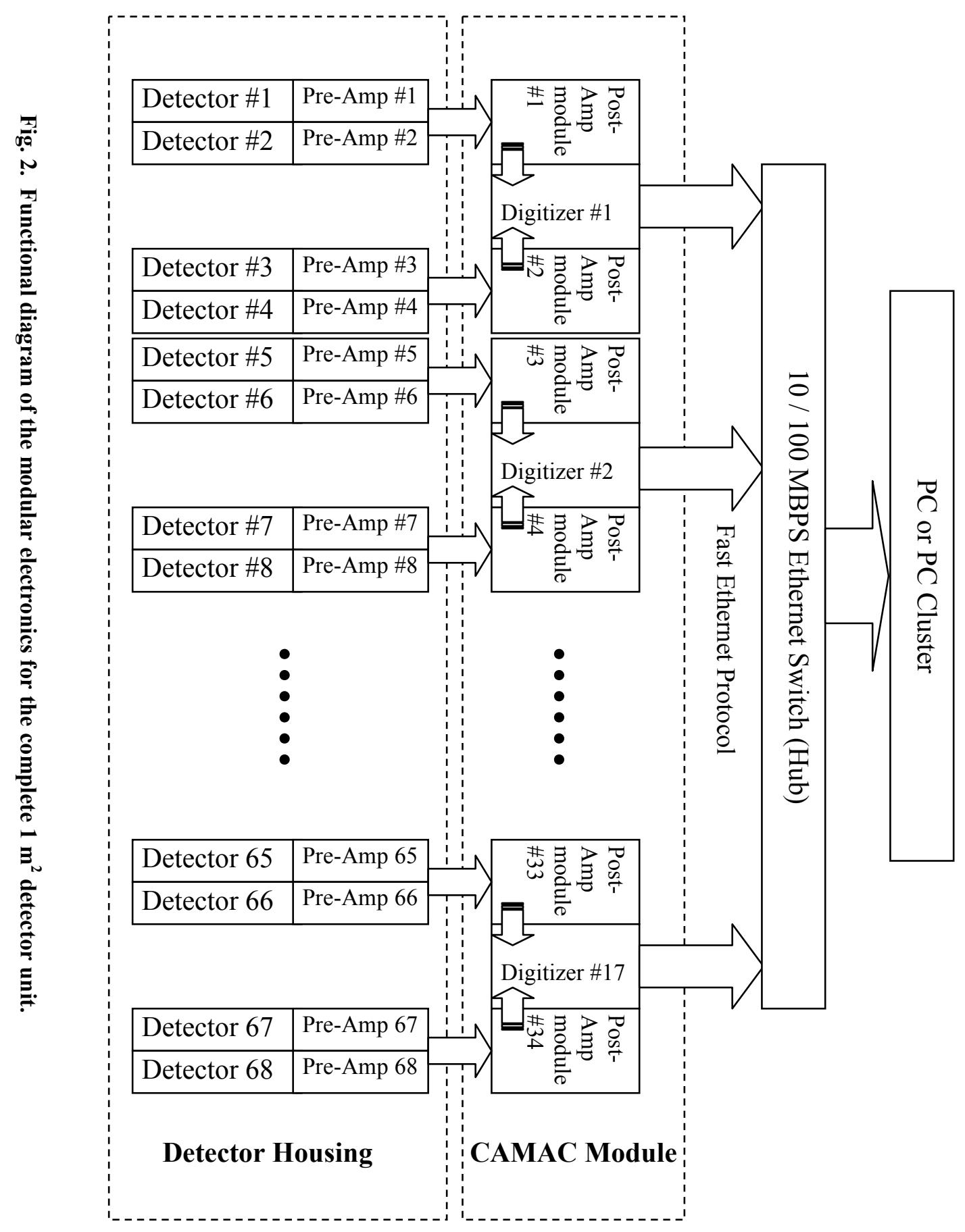




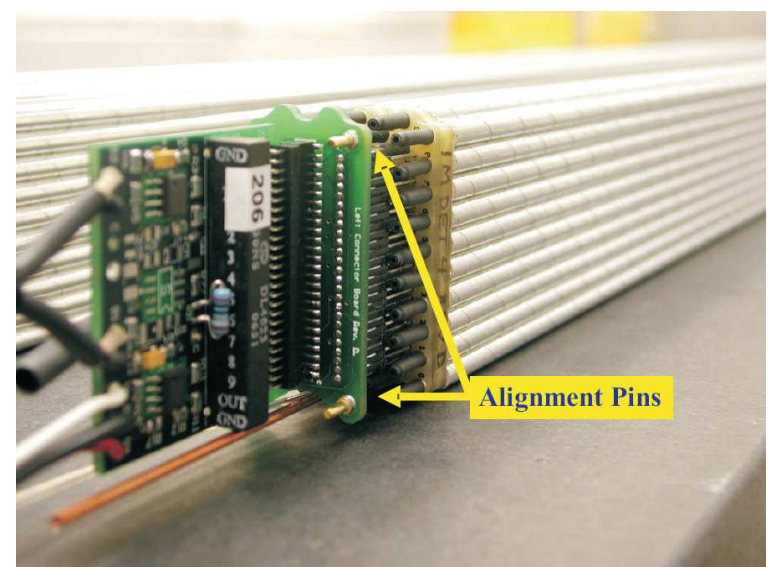

Fig. 3. A completed 1 meter module with amplifier boards attached. Gold PC board alignment pins can be seen mounted to the amplifier connector board slightly left of center in the image. These pins guide detector positioning into the panel upper and lower alignment structures.

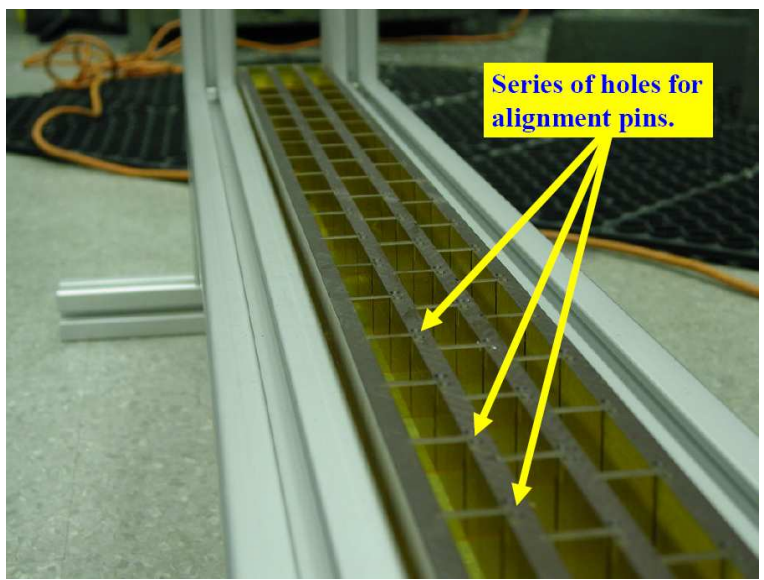

Fig. 4. The alignment structure shown mounted in the bottom of the detector panel housing is machined from Ultem1000. This structure allows precise detector positioning and support. 


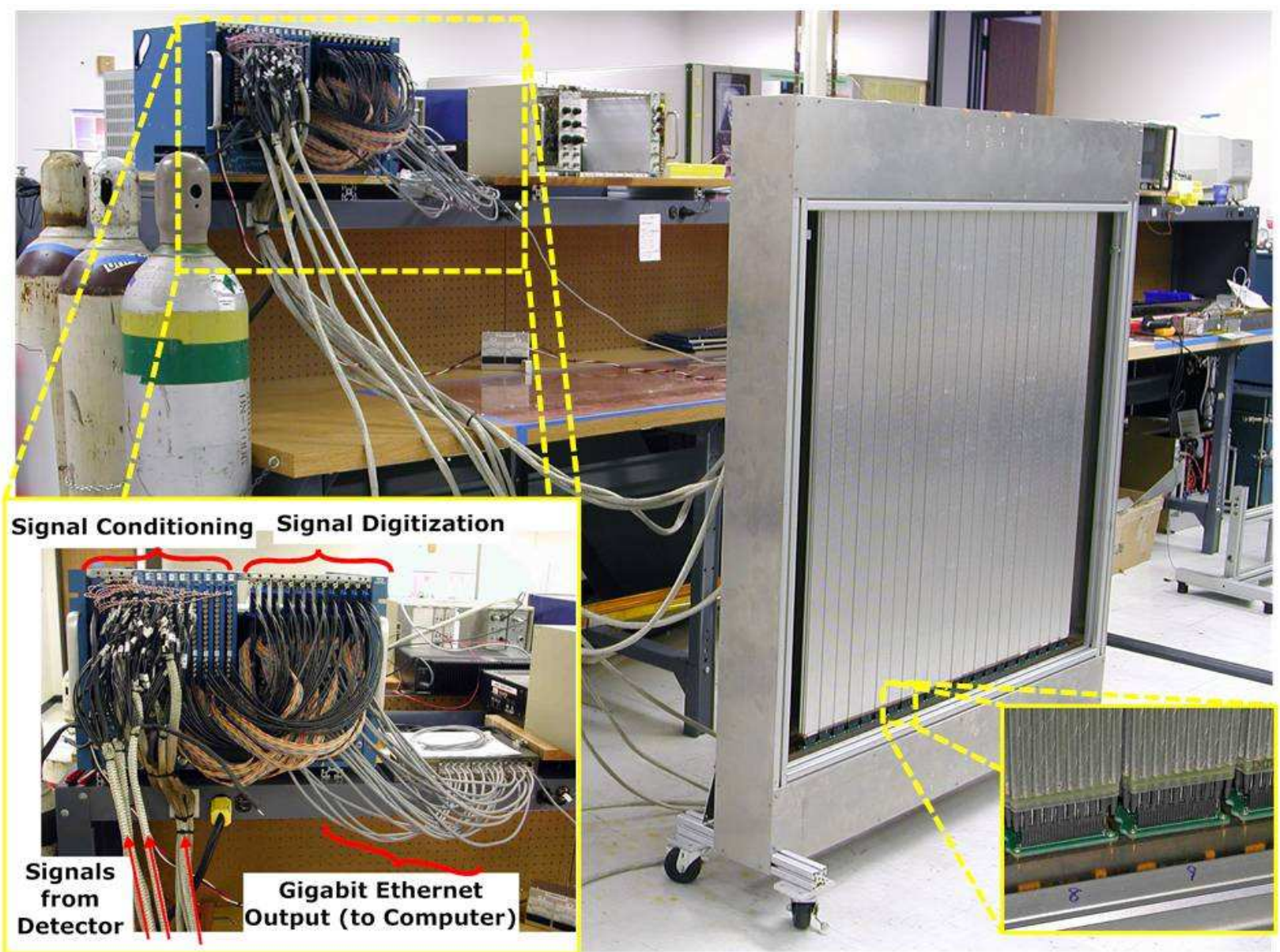

Fig. 5. Shown (right) is the custom-designed housing populated with 22 detector modules installed (bottomright insert). The electronic readout of signals (bottom-left insert) provides all connections to power, highvoltage, signal data input, digitization, and computer output. A total of 22 modules can be installed, forming a sensitive area of 1 square meter. 


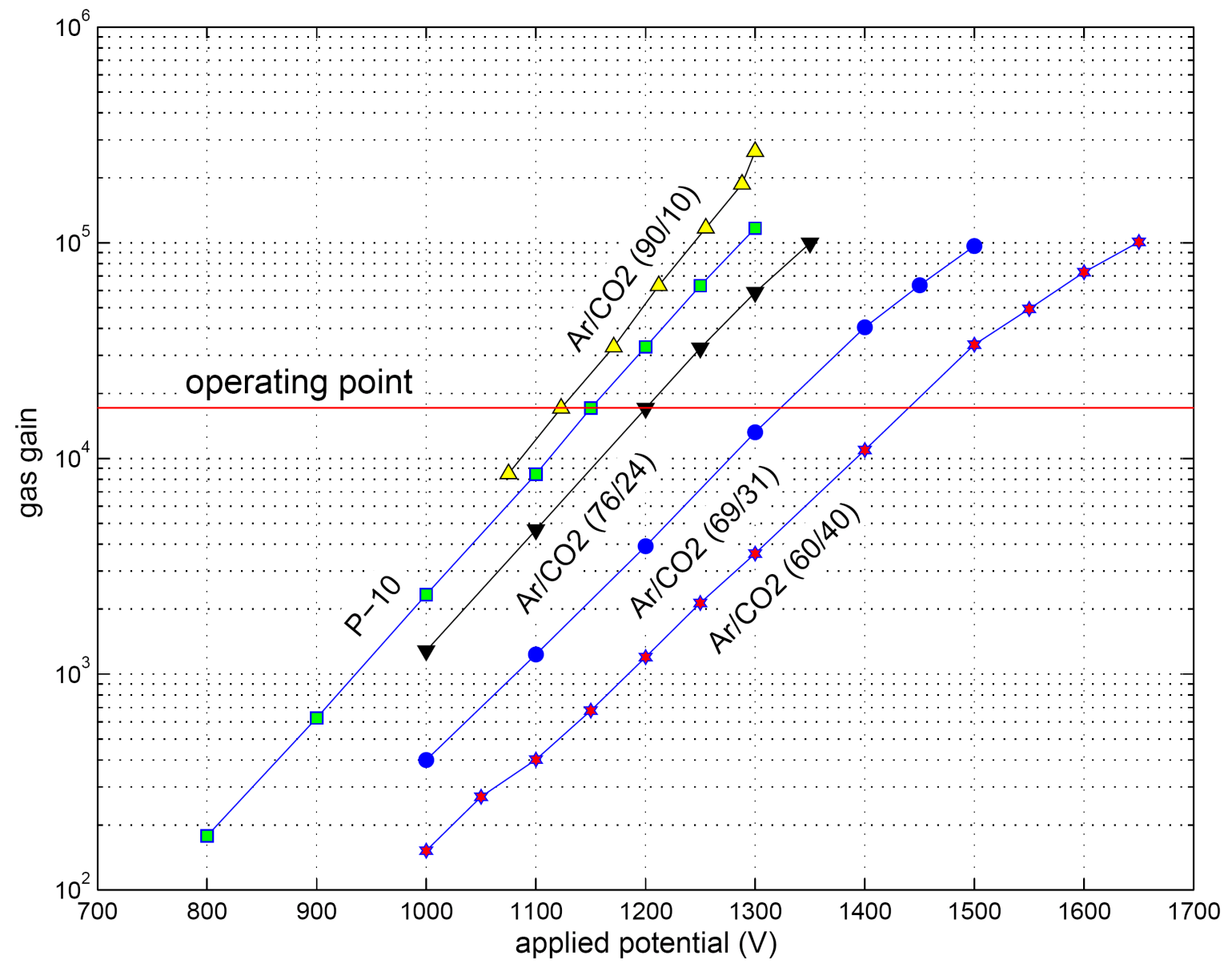

Fig. 6. Gas gain comparison between various $\mathrm{Ar} / \mathrm{CO}_{2}$ mixtures and P-10. 


$$
\text { P-10 }
$$
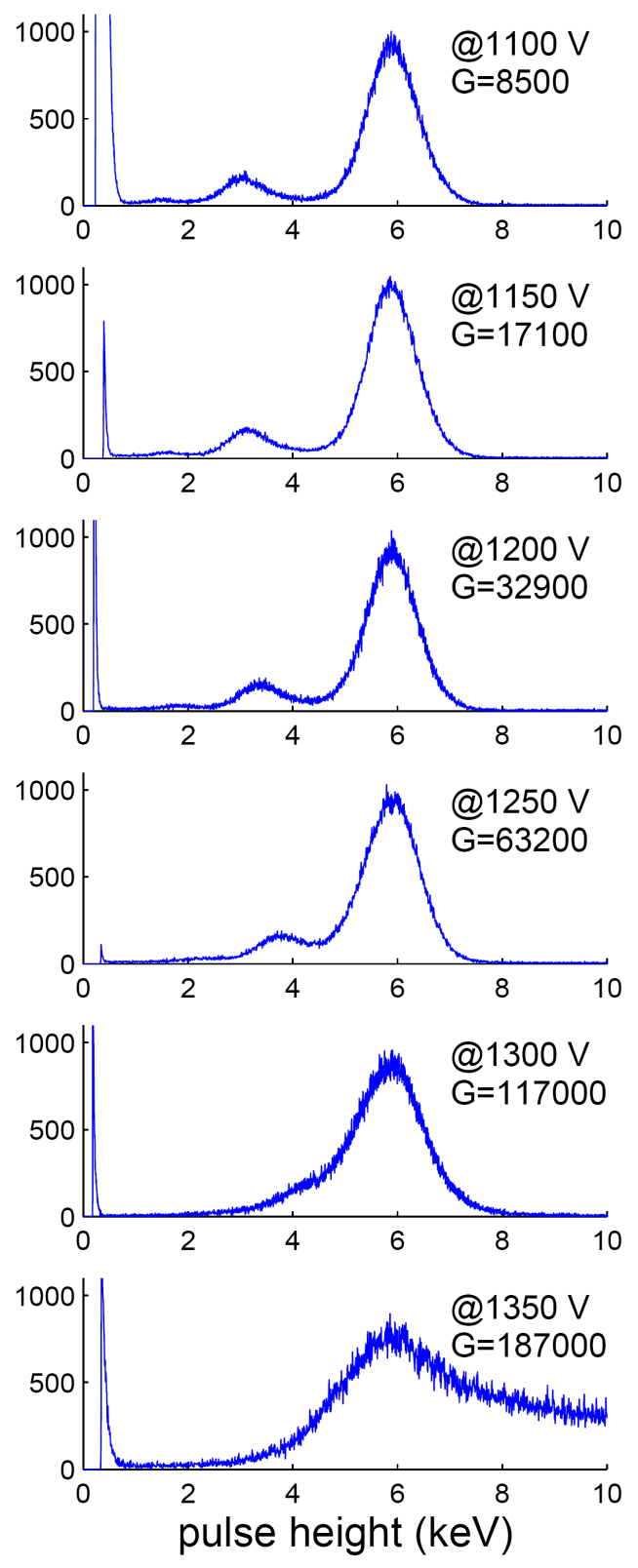

$\mathrm{Ar} / \mathrm{CO}_{2}(82 / 18)$
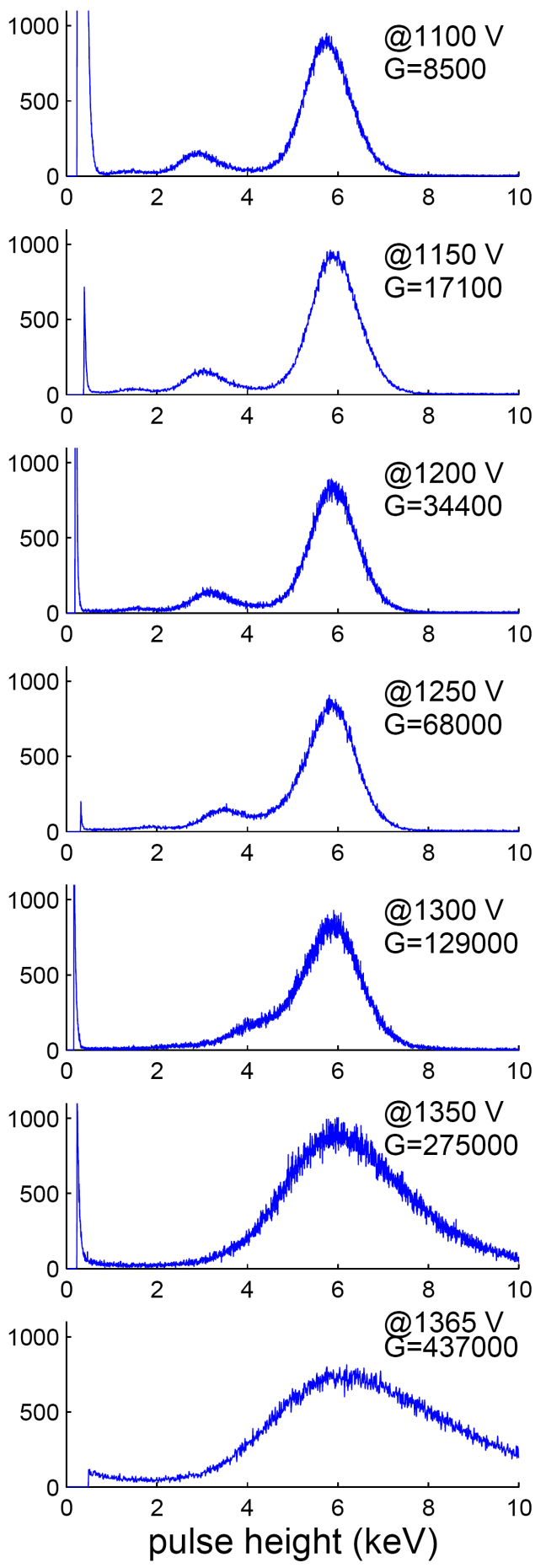

Fig. 7. Fe-55 energy spectra collected in a straw detector operated with two different gas mixtures, P-10 on the left column, and $\mathrm{Ar} / \mathrm{CO}_{2}(82 / 18)$ on the right column. The applied potential and gas gain are indicated in each panel. 


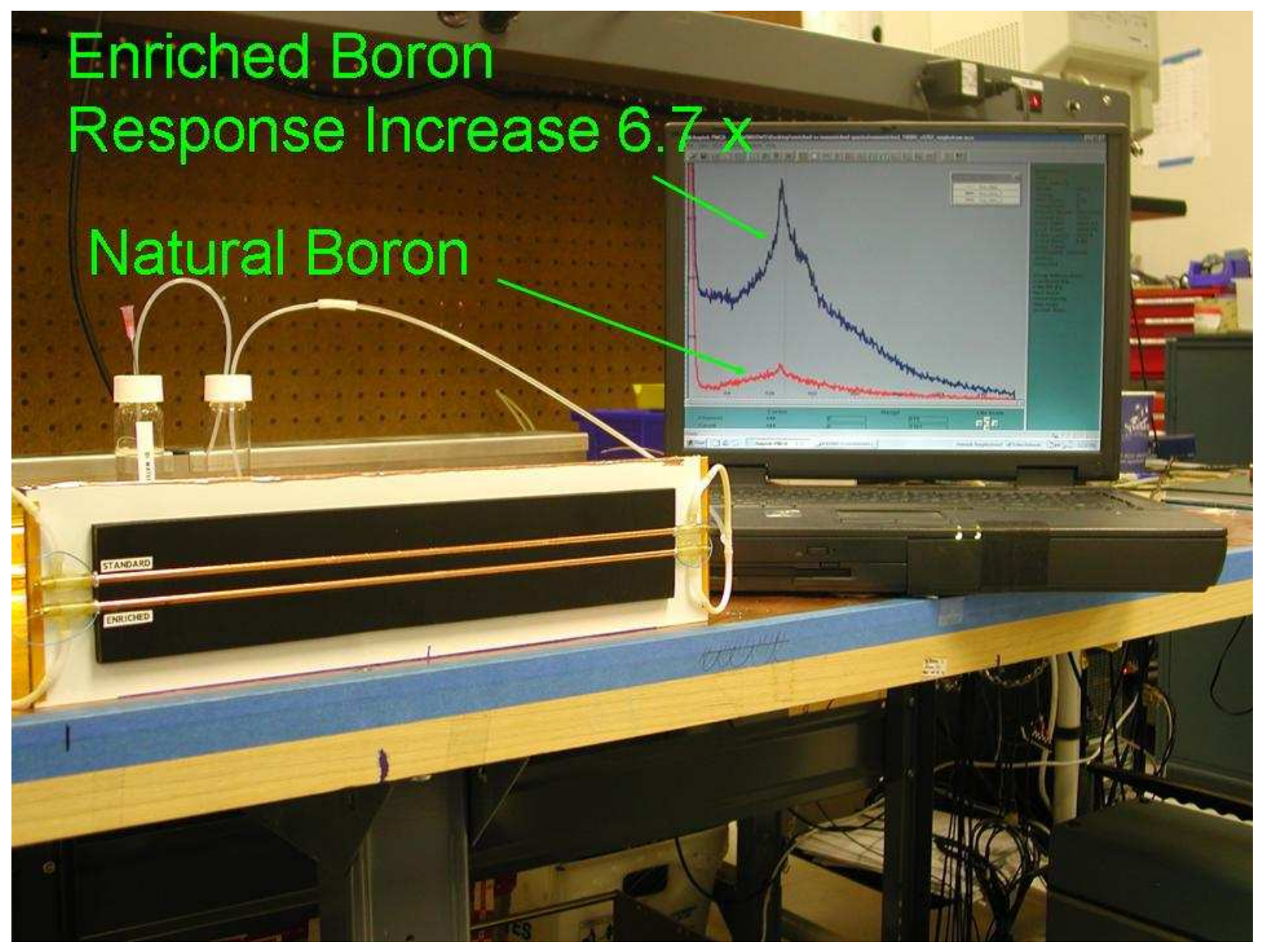

Fig. 8. Single enriched and natural boron coated straw detectors were operated within the same thermal neutron flux using the same set of electronics. The spectra show an enriched boron response increase of 6.7 times that of the natural boron coated straw. 

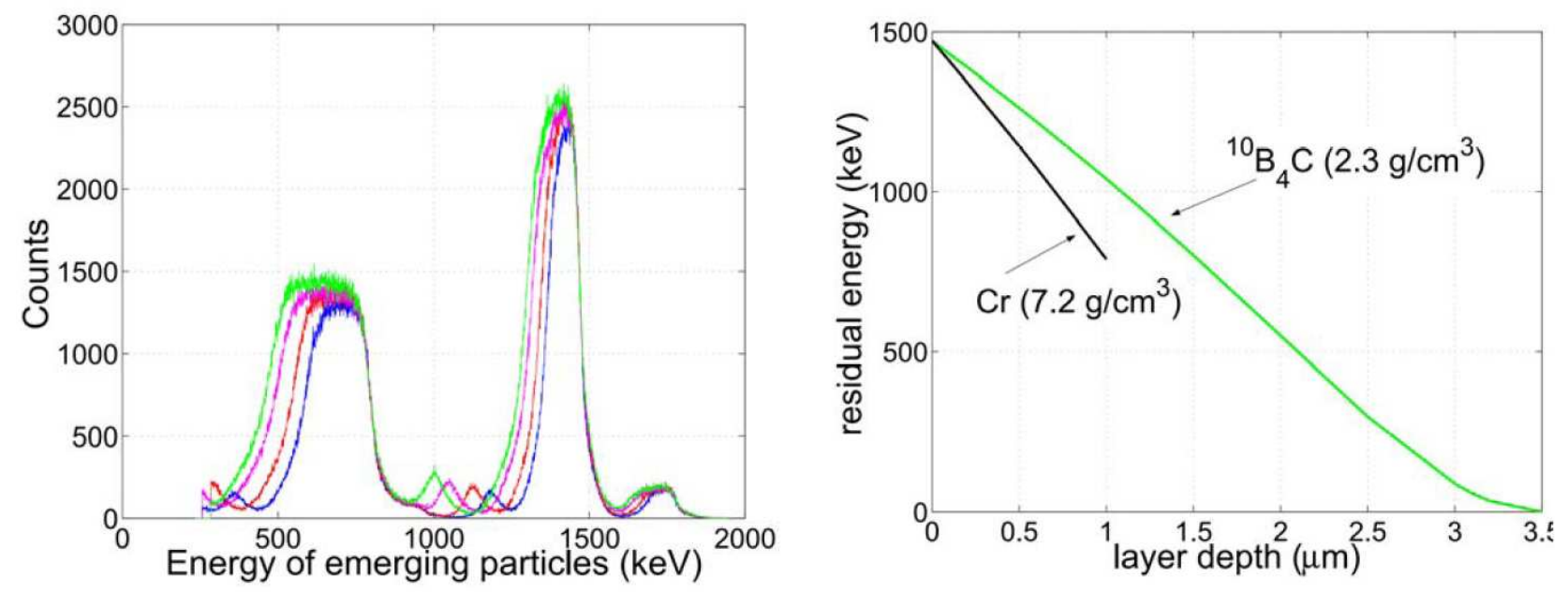

Fig. 9. left, Energy distribution of alphas and Li ions escaping a ${ }^{10} \mathrm{~B}_{4} \mathrm{C}$-coated foil sample. Multiple lines correspond to different samples; right, Energy loss of alpha particles as they travel inside a thin layer of boron carbide, and chromium. 

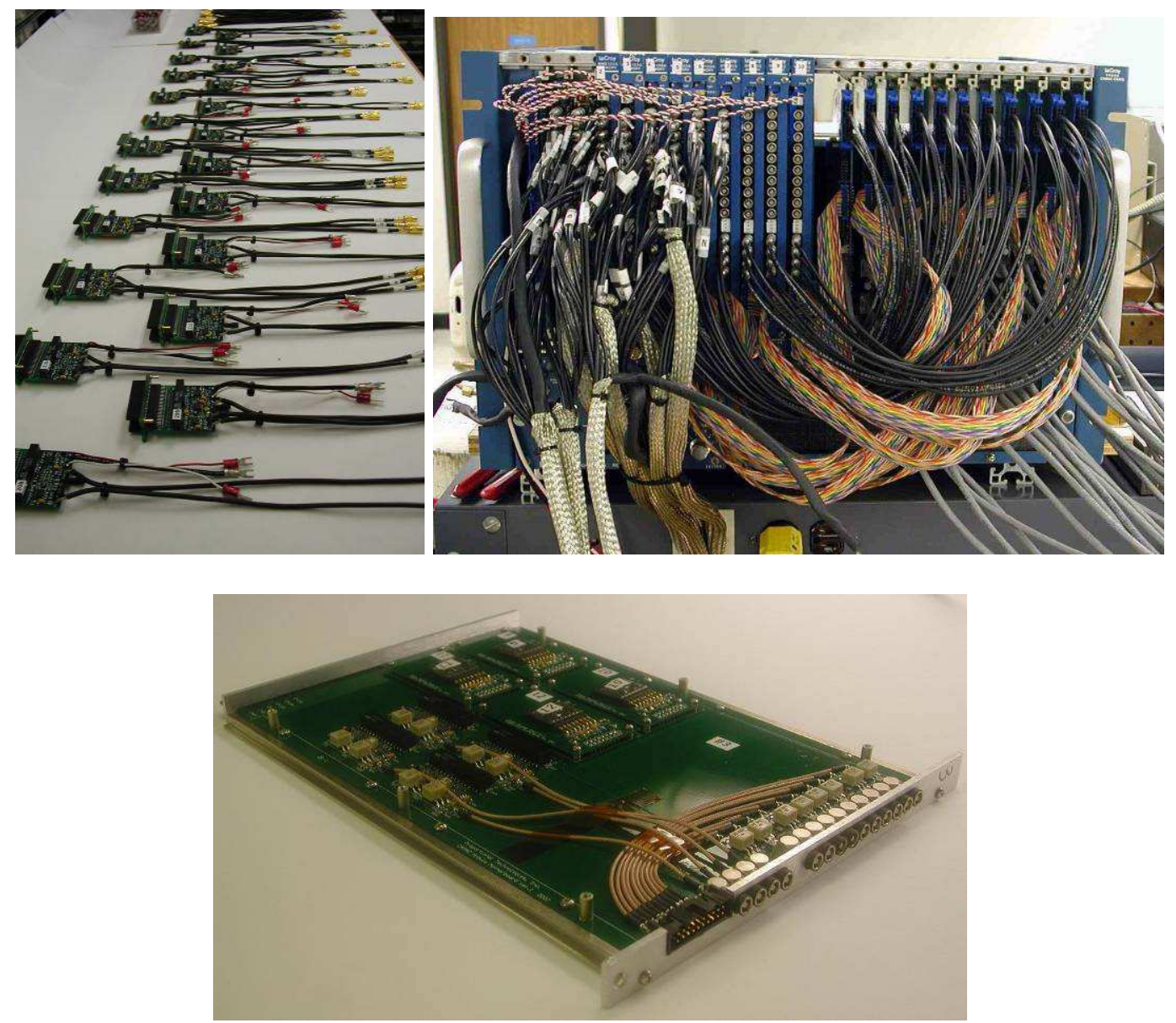

Fig. 10. Shown are (top left) completed pre-amplifiers awaiting installation onto the ends of the detector modules; (top right) 22 signal conditioning modules along with signal digitization modules in a CAMAC crate; and (bottom) a CAMAC module for signal conditioning. 


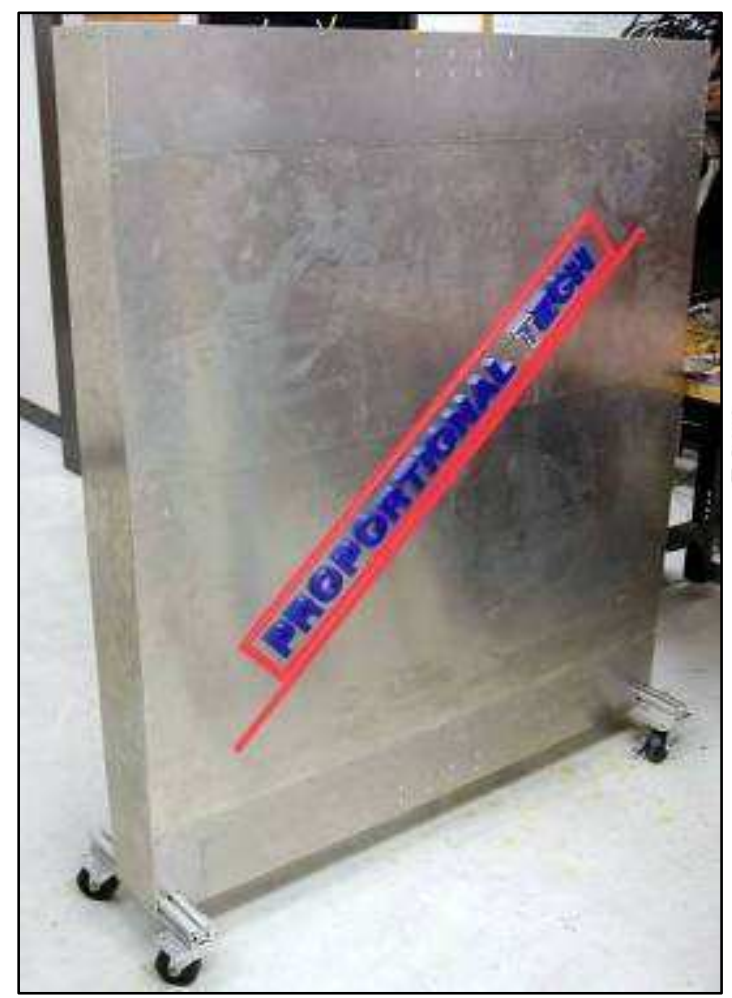

(a)

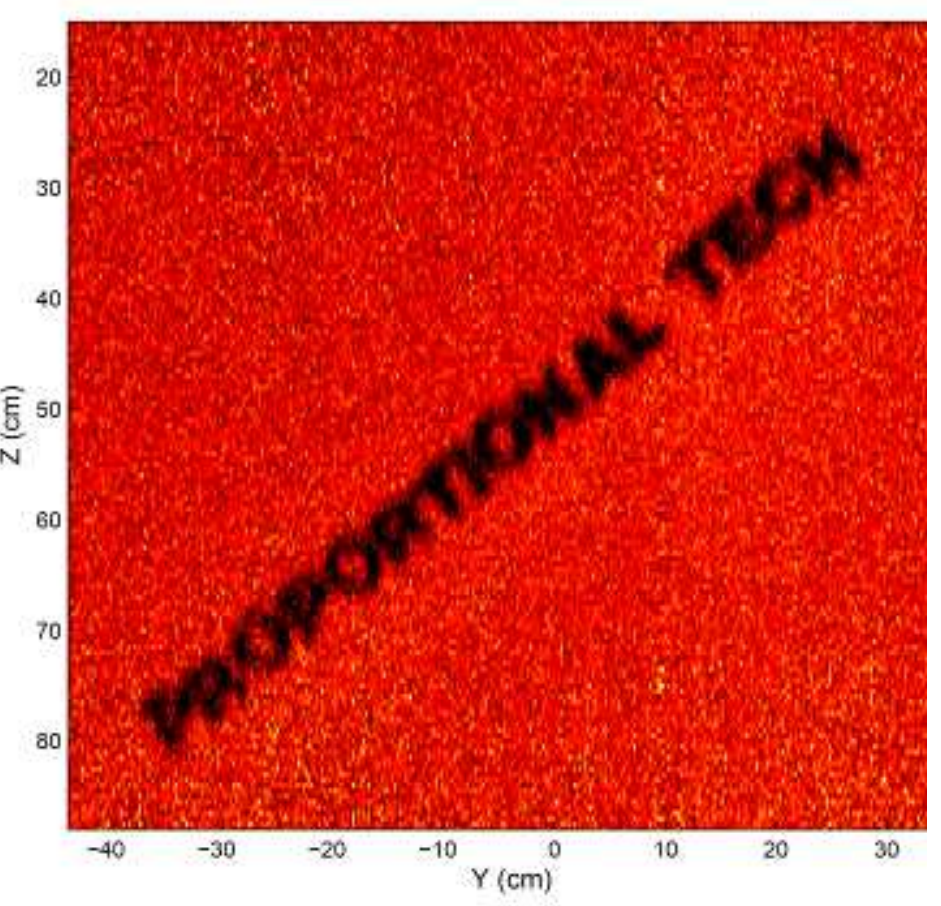

(b)

Figure 11. Phantom study using acrylic letters. (a) The letters $(9.5 \mathrm{~mm}$ thick, $55 \mathrm{~mm}$ tall) were attached directly onto the front face of the detector. Neutrons originated from a moderated ${ }^{252} \mathrm{Cf}$ neutron source 2.2 meters away; (b) Image constructed with 20 million neutron events. 


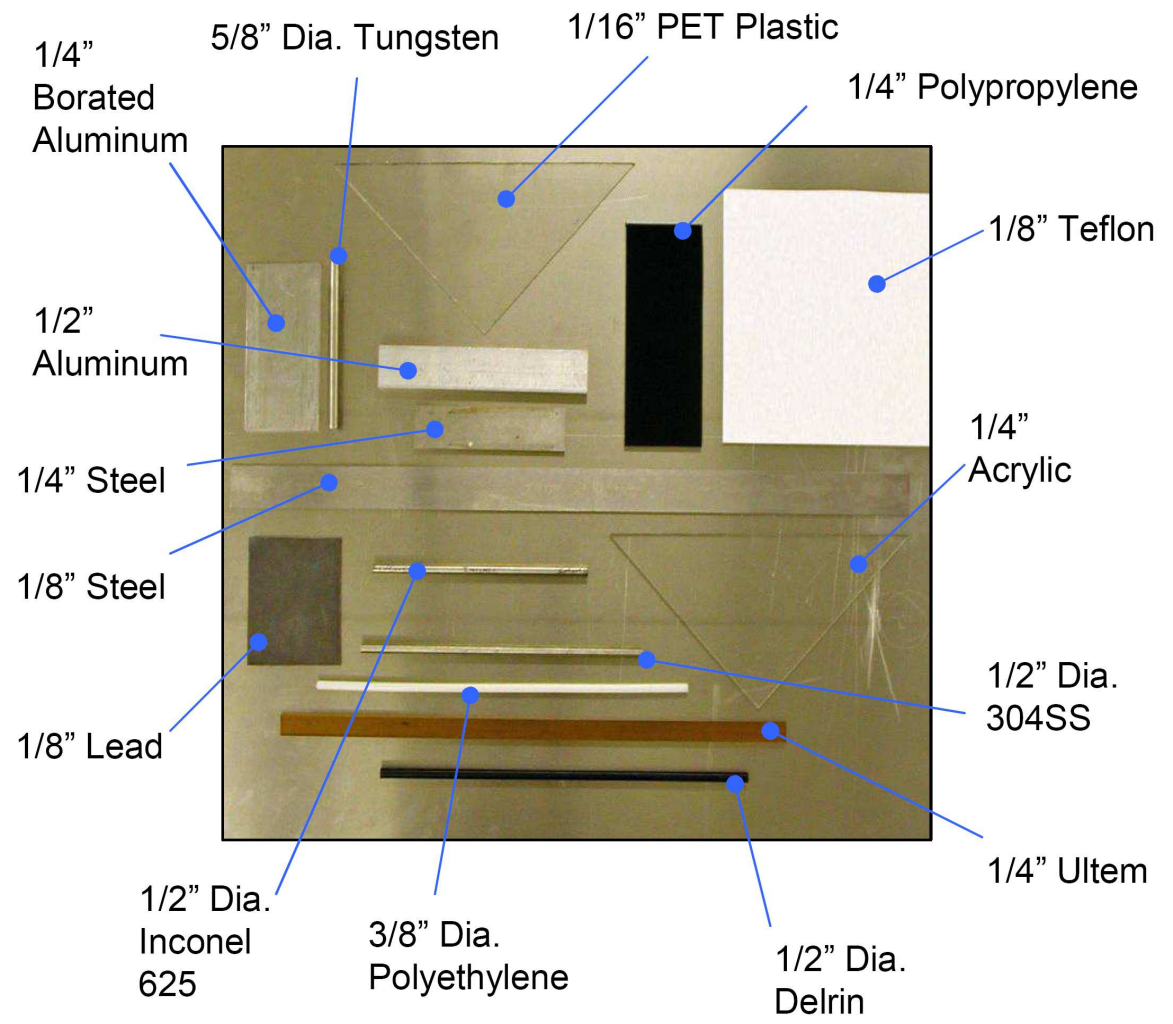

(a)

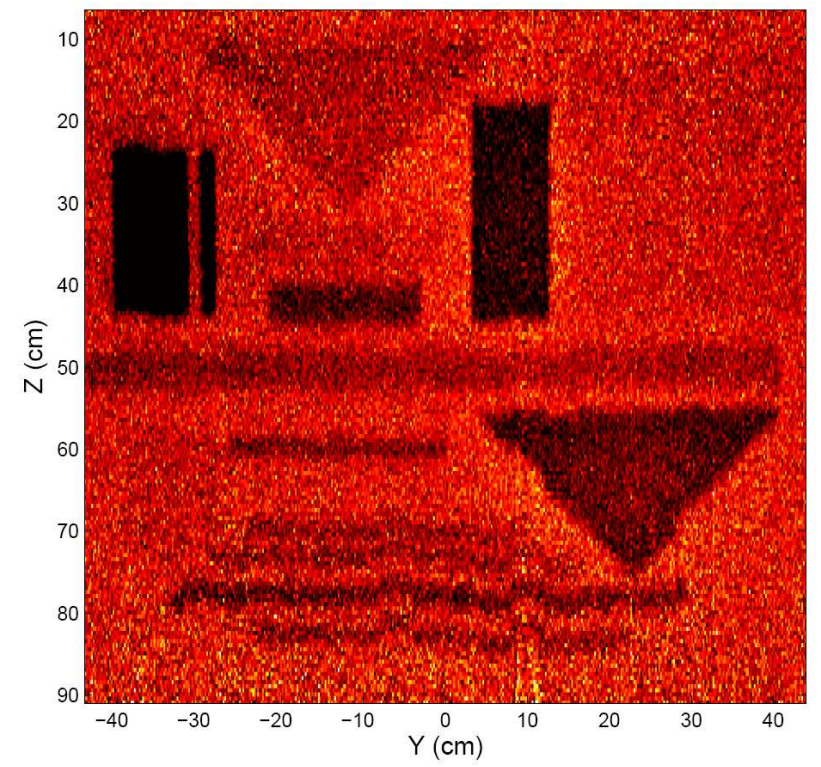

(b)

Figure 12. Random objects image (a). Objects of different shape, composition and thickness were used (numbers represent thickness in inches); (b) Image constructed with 13 million neutron events. 